\title{
Compact integrated depolarizer for interferometric fiber optic gyroscopes
}

\author{
Lixia Li \\ University of Alabama - Huntsville \\ Jaime Cardenas \\ Jianhua Jiang \\ Gregory P. Nordin \\ Brigham Young University - Provo, nordin@byu.edu
}

Follow this and additional works at: https://scholarsarchive.byu.edu/facpub

Part of the Electrical and Computer Engineering Commons

\section{Original Publication Citation}

Lixia Li, Jaime Cardenas, Jianhua Jiang and G. P. Nordin, "Compact integrated depolarizer for IFOG," Opt. Eng. 45, 5562 (26)

\section{BYU ScholarsArchive Citation}

Li, Lixia; Cardenas, Jaime; Jiang, Jianhua; and Nordin, Gregory P., "Compact integrated depolarizer for interferometric fiber optic gyroscopes" (2006). Faculty Publications. 1349.

https://scholarsarchive.byu.edu/facpub/1349 accepted for inclusion in Faculty Publications by an authorized administrator of BYU ScholarsArchive. For more information, please contact ellen_amatangelo@byu.edu. 


\section{Compact integrated depolarizer for interferometric fiber optic gyroscopes}

\author{
Lixia Li \\ Jaime Cardenas \\ Jianhua Jiang \\ Gregory P. Nordin, MEMBER SPIE* \\ University of Alabama in Huntsville \\ Nano and Micro Devices Center \\ Huntsville, Alabama 35899 \\ E-mail: nordin@ee.byu.edu
}

\begin{abstract}
We propose an integrated waveguide depolarizer for use in interferometric fiber optic gyroscopes (IFOGs) with single-mode fiber coils. The integrated waveguide depolarizer is based on a Mach-Zender interferometer with polarizing beamsplitters. A waveguide polarizing beamsplitter is designed using multiple air trench structures oriented at the Brewster angle. We also analyze the effect of component imperfections on the degree of polarization achievable with an integrated waveguide depolarizer. (c) 2006 Society of Photo-Optical Instrumentation Engineers. [DOI: $10.1117 / 1.2205827]$
\end{abstract}

Subject terms: interferometric fiber optic gyroscope; gyroscope; depolarizer; polarization; integrated optics; guided waves; optical devices; waveguides; planar lightwave circuit.

Paper 050379R received May 11, 2005; revised manuscript received Sep. 28 , 2005; accepted for publication Oct. 13, 2005; published online May 24, 2006.

\section{Introduction}

Hybrid interferometric fiber optic gyroscopes (IFOGs) were originally developed to realize systems that were more rugged than what could be achieved with bulk optics in combination with an optical fiber sensing coil. ${ }^{1}$ The typical configuration initially consisted of a light source, an all fiber $2 \times 2$ coupler, an optoelectronic integrated circuit (OEIC), and a polarization maintaining (PM) fiber coil (see Fig. 1). To achieve high performance, the coil was made of PM fiber, which is much more expensive than single-mode (SM) fiber. This, in turn, made IFOGs costly. While the integration of several components into the OEIC (polarizer, splitter, and phase modulators) created some cost reduction, the cost was still dominated by the PM fiber coil. Thus, Bohm et al. proposed the use of a depolarizer and a SM fiber coil as an alternative. ${ }^{2}$ The typical layout for an IFOG with an SM fiber coil is depicted in Fig. 2. The depolarizers usually used in IFOGs consist of two pieces of PM fiber spliced with their slow axes at a $45-\mathrm{deg}$ angle. ${ }^{2}$ While these depolarizers can be readily made using modern automatic alignment fusion splicers, the slow axis of the PM fiber must be aligned to the polarization state axis of the OEIC. In this paper we propose, design, and analyze a depolarizer that can be integrated into the OEIC, which eliminates the labor-intensive and costly step of PM fiber alignment to the OEIC and achieves greater system integration.

Many different kinds of depolarizers have been proposed in the literature. ${ }^{3-7}$ Among them are all fiber ${ }^{3-5}$ or bulk optics approaches ${ }_{7}^{6,7}$ broadband $d^{3,4}$ or laser ${ }^{5-7}$ source depolarizers, and active ${ }^{7}$ or passive $e^{3-6}$ methods. Fiber Lyot depolarizers can be made by splicing two pieces of PM fiber of the appropriate length whose fast axes are at $45 \mathrm{deg}$ with respect to each other. ${ }^{3}$ This depolarizer requires a

\footnotetext{
*Present address: 458 Clyde Building, Brigham Young University, Provo, Utah 84602.

0091-3286/2006/\$22.00 @ 2006 SPIE
}

broadband source. Another fiber depolarizer is based on a Mach-Zender interferometer. ${ }^{4}$ It operates by splitting the incident beam into two polarized components. One of these components is delayed to make the two beams incoherent. Finally, they are recombined to get depolarized light. Depending on the fiber delay length, broadband and laser sources can be depolarized. To the authors' knowledge, a passive depolarizer integrated onto a planar lightwave circuit has not been proposed in the literature.

\section{Methodology}

In this section, we discuss the approach to depolarize light followed by the design of the waveguide components. Additionally, we analyze the proposed depolarizer to obtain a general expression that shows the effects of imperfect components on the attainable degree of polarization.

\subsection{Depolarizer Approach}

The Stokes vectors of two beams can be represented graphically using the Poincaré sphere, ${ }^{8}$ as shown in Fig. 3. In general, when two incoherent, arbitrarily polarized beams, $S_{a}$ and $S_{b}$, overlap, their Stokes vectors add. If these vectors lay on opposite sides of the sphere, as in Fig. 3, the result of their addition is a point at the center of the sphere $O$, which represents completely unpolarized light and is denoted by the Stokes vector $\boldsymbol{S}_{o}^{T}=\left[\begin{array}{llll}1 & 0 & 0 & 0\end{array}\right]^{T}$.

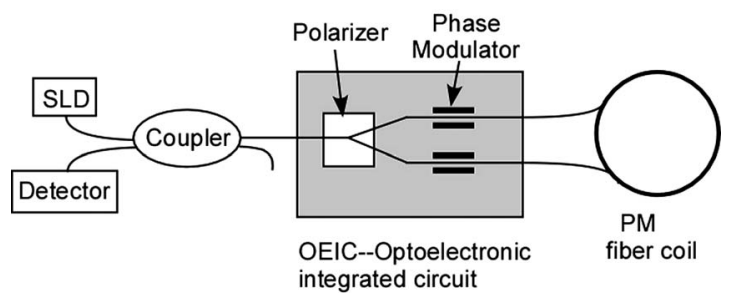

Fig. 1 Hybrid IFOG schematic in which an OEIC has the polarizer, splitter, and phase modulators integrated and the sensing coil is made with PM fiber. 


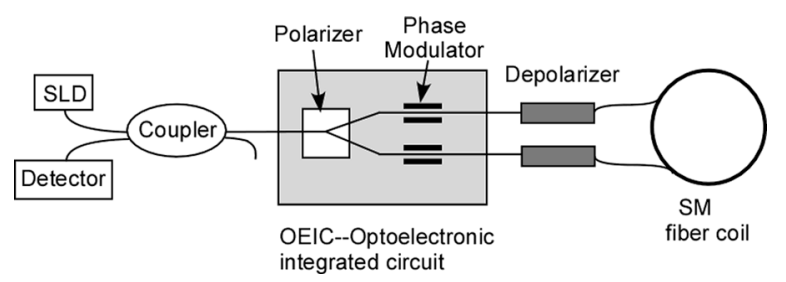

Fig. 2 Depolarized IFOG configuration.

This process for depolarizing light ${ }^{6}$ can be implemented as illustrated in Fig. 4 in which incident linearly polarized TM (electric field in the plane of the figure) light is transformed into an elliptical state with equal TE (electric field out of the plane of the figure) and TM components by the polarization rotator. A polarization beamsplitter (PBS) separates the two polarization components into different paths. The path length difference between them is selected to be greater than the source's coherence length. For the source used in the experimental demonstration discussed in Sec. 3.1, the path length difference is only $265 \mu \mathrm{m}$, [see Fig. 9(b) in Sec. 3]. Thus, when these two, equal-intensity and incoherent beams recombine, their Stokes vectors add to generate unpolarized light. (Note that the depolarizer can be designed to operate with other incident polarization states by appropriately designing the polarization rotator.)

\subsection{Waveguide Component Design}

The critical waveguide components of the integrated depolarizer are the polarization rotator, the PBSs, and the mirrors. Several different approaches for the polarization rotator may be found in the literature. ${ }^{9-12}$ In this paper, we address the detailed design of waveguide PBSs and mirrors. We assume a ridge waveguide for the integrated depolarizer, as shown in Fig. 5 in which the core is polyimide PI-2525 and the upper and lower cladding layers are NOA 71 (Norland Optical Adhesive 71). The refractive index for the core is 1.656 and 1.640 for TM and TE polarizations, respectively. For the cladding, the refractive index is 1.548 for TM and 1.547 for TE polarization. The waveguide geometry is designed using Fimmwave. ${ }^{13}$ For SM propagation at $\lambda_{0}=1.33 \mu \mathrm{m}$, the waveguide dimensions are $W$ $=3.5 \mu \mathrm{m}, H=0.7 \mu \mathrm{m}$, and $H_{1}=2.3 \mu \mathrm{m}$. For simulation

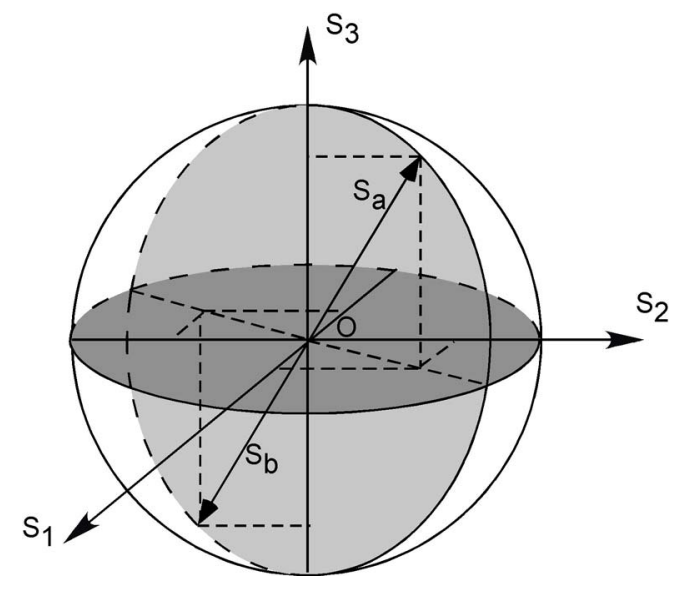

Fig. 3 Poincare sphere illustration of depolarizer approach.

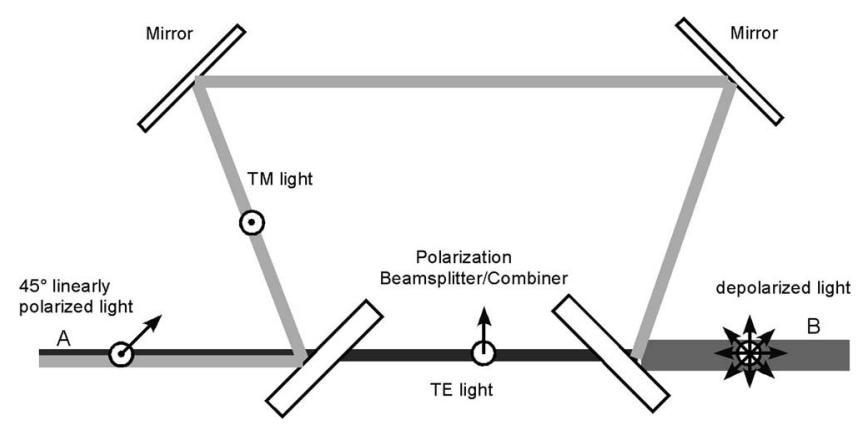

Fig. 4 Component layout of depolarizer.

purposes, the 3-D structure is transformed into a 2-D slab waveguide using the effective index method (EIM). The result is a slab waveguide with a width of $3.5 \mu \mathrm{m}$ and core and cladding refractive indices of 1.6302 and 1.6251 for TM polarization and 1.6464 and 1.6414 for TE polarization, respectively.

A key component in the integrated depolarizer is the waveguide PBS (WPBS). We design this component by making a dielectric mirror oriented at Brewster angle incidence such that TE polarization is transmitted and TM is reflected. In our case, the Brewster angle, which is defined by a polymer-air interface with refractive indices of 1.6414 (the waveguide effective refractive index for TE polarization) and one, is approximately $31.35 \mathrm{deg}$. For TM polarization, we use a multilayer structure composed of etched air trenches to create a high-reflectivity element.

The main parameters for the design of the highreflectivity mirror are the thickness and number of layers. The well-known analytical equation ${ }^{14}$ to calculate each layer's thickness is $n t \cos \theta=M\left(\lambda_{0} / 4\right)$, where $\lambda_{0}=1.33 \mu \mathrm{m}$; and $n, t$, and $\theta$ are the refractive index, layer thickness, and propagation angle in each alternate layer; and $M$ is any odd integer. Thicker layers can make fabrication more feasible. Thus, taking $M=1$ for the air layer and $M=3$ for the cladding layer, the thicknesses are 0.62 and $0.72 \mu \mathrm{m}$, respectively. Approximating the waveguide mode as a plane wave, five air layers are necessary to achieve over $99 \%$ reflection efficiency for TM polarization.

While using five layers results in a highly efficient mirror, our simulations show that a five-layer structure decreases the transmittance for the TE polarized waveguide mode even with Brewster angle incidence. This occurs because the Brewster angle of 31.35 deg is calculated based on a plane wave approximation. The waveguide mode can

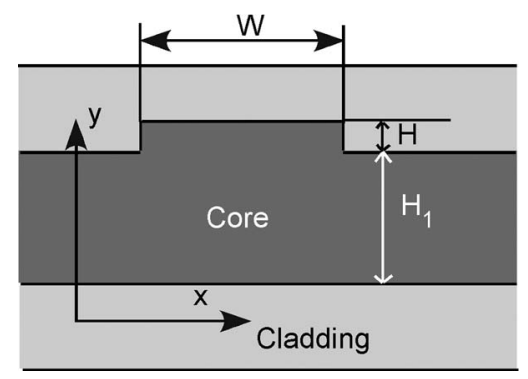

Fig. 5 A 3-D cross section of gyro waveguide. 
be decomposed into plane wave components, and most of these components will not be incident at precisely the Brewster angle. Instead, they will undergo both transmission and reflection at each air-polymer interface. The more layers the structure has, the worse the degradation for TE polarization. However, selecting fewer layers decreases the reflection of the TM polarization. We have found that a reasonable balance is achieved with three layers. Figure 6 shows finite difference time domain ${ }^{15}$ (FDTD) simulation results for a three-layer PBS computed with a Yee cell grid size of $\lambda_{0} / 60$. The reflection for TM-polarized light is $97 \%$ and the transmission for TE is $92.8 \%$. The reflection for TE and transmission for TM are 0.24 and $1.9 \%$, respectively. These small quantities represent cross-talk and will affect the achievable degree of polarization (DoP).

The only requirement for the mirrors on the longer path for TM polarization is a high reflectivity. With the configuration of Fig. 4, the mirror also has Brewster angle incidence. Based on the preceding analysis, five layers will give us a high-performance mirror for TM polarization. FDTD simulations show a bend efficiency of $98.8 \%$ for TM polarization, while a reflectivity of only $5.9 \%$ for TE (see Fig. 7).

\subsection{DoP Error Analysis}

If ideal components are considered for the integrated waveguide depolarizer, the resulting DoP will be limited only by the residual coherence of the two beams. However, the mirrors will not reflect $100 \%$ of the incident light and the PBS will not reflect one polarization state perfectly while completely transmitting the other (i.e., there will be some leakage of unwanted polarization components into both legs of the ring.) Similarly, the output of the polarization rotator will not have exactly equal TE and TM components. All of these imperfections will affect the DoP. In our particular application, we require a DoP of 0.05 or less.

To account for the effect of component imperfections, we use Jones and Stokes vectors to analyze the integrated depolarizer. We can write the Jones vector for the output of the polarization rotator (refer to Fig. 4) as

$\hat{\boldsymbol{e}}_{B}=\left(\begin{array}{c}\kappa \\ \Delta e^{j \delta}\end{array}\right)$,

where $\Delta$ and $\kappa$ are the fractional amounts of TM and TE polarized light, respectively, and $\Delta^{2}+\kappa^{2}=1$. (Note that in all cases, the $x$ and $y$ Jones vector components refer to TE and TM electric fields.)

After the first PBS, the reflected polarization state will be given by

$\hat{\boldsymbol{e}}_{D}=\left(\begin{array}{c}\varepsilon_{D} \kappa \\ I_{R} \Delta e^{j \delta}\end{array}\right)$,

where $I_{R}$ and $\varepsilon_{D}$ are the reflected portions of TM and TE polarized light, respectively, and $I_{R}^{2}+\varepsilon_{D}^{2}=1$. Similarly, the transmitted polarization state will be given by

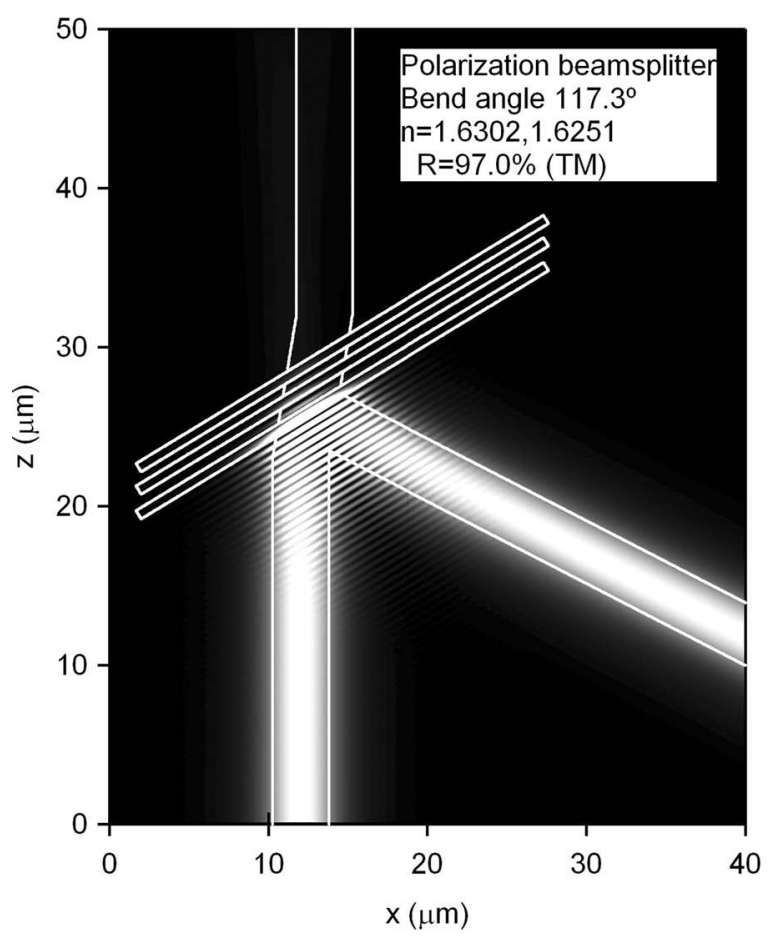

(a)

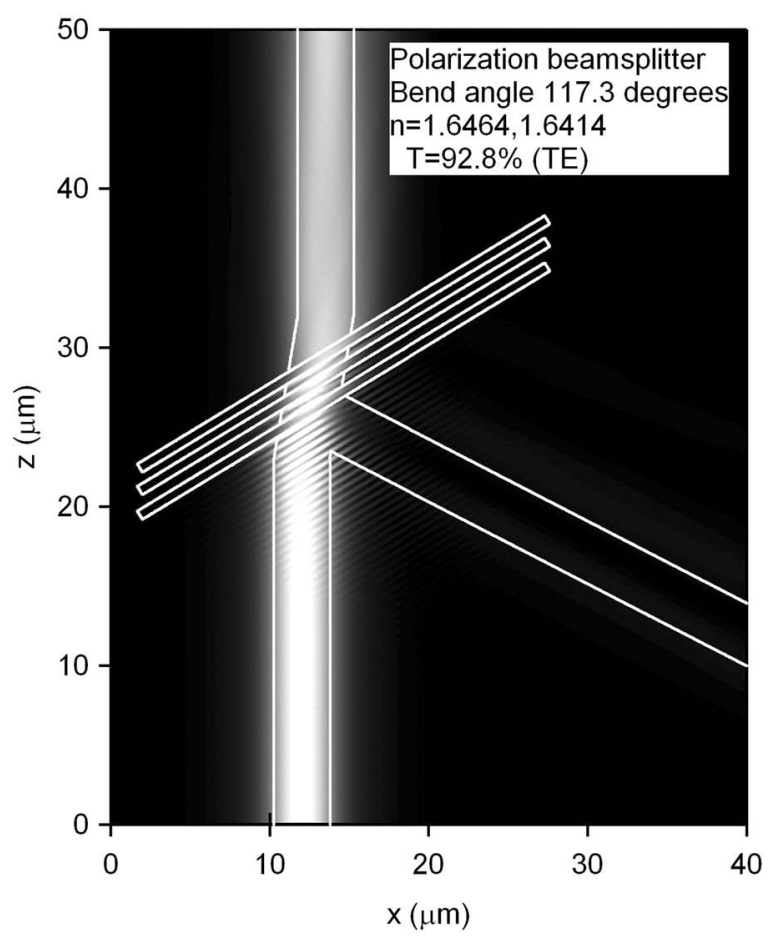

(b)

Fig. 6 WPBSs for (a) TM incidence and (b) TE incidence.

$\hat{\boldsymbol{e}}_{C}=\left(\begin{array}{c}\kappa I_{t} \\ \boldsymbol{\varepsilon}_{C} \Delta e^{j \delta}\end{array}\right)$,

where $\varepsilon_{C}$ and $I_{t}$ are the transmitted fractions of TM and TE polarized light, respectively, and $I_{t}^{2}+\varepsilon_{C}^{2}=1$.

The mirrors are designed to provide maximum reflectiv- 


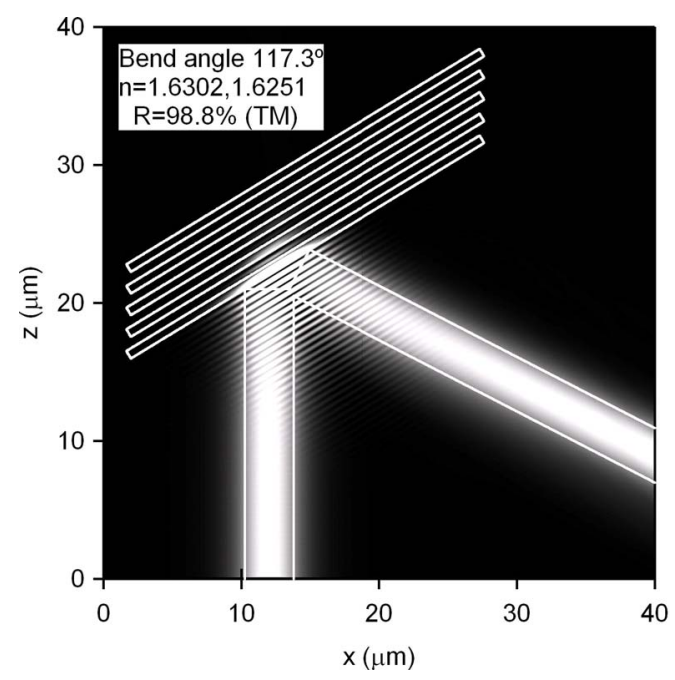

Fig. 7 Waveguide mirror (TM incidence).

ity for the expected polarization component in the longer arm. The leaked component will not undergo an efficient reflection. The Jones matrix representing the mirrors is given by

mirror $=\left(\begin{array}{cc}\varepsilon_{M} & 0 \\ 0 & I_{M}\end{array}\right)$.
Using these vectors and the mirrors' Jones matrix, and after converting the resulting Jones vectors into Stokes vectors, it can be shown that the Stokes vector describing the light going around the longer path is

$S_{E r}=\left(\begin{array}{c}\kappa^{2} \varepsilon_{D}^{4} \varepsilon_{M}^{4}+I_{R}^{4} I_{M}^{4} \Delta^{2} \\ \kappa^{2} \varepsilon_{D}^{4} \varepsilon_{M}^{4}-I_{R}^{4} I_{M}^{4} \Delta^{2} \\ 2 I_{R}^{2} I_{M}^{2} \kappa \varepsilon_{D}^{2} \varepsilon_{M}^{2} \Delta \cos \delta \\ -2 I_{R}^{2} I_{M}^{2} \kappa \varepsilon_{D}^{2} \varepsilon_{M}^{2} \Delta \sin \delta\end{array}\right)$,

and similarly for the light transmitted through the short path,

$S_{E t}=\left(\begin{array}{c}\kappa^{2} I_{t}^{4}+\varepsilon_{C}^{4} \Delta^{2} \\ \kappa^{2} I_{t}^{4}-\varepsilon_{C}^{4} \Delta^{2} \\ 2 I_{t}^{2} \kappa \varepsilon_{C}^{2} \Delta \cos \delta \\ -2 I_{t}^{2} \kappa \varepsilon_{C}^{2} \Delta \sin \delta\end{array}\right)$.

Given that the Stokes vector is

$S=\left(\begin{array}{c}s_{0} \\ s_{1} \\ s_{2} \\ s_{3}\end{array}\right)$,

and that the DoP is $\left(s_{1}^{2}+s_{2}^{2}+s_{3}^{2}\right)^{1 / 2} / s_{0}$, it can be shown that after adding the Stokes vectors the DoP in the output waveguide is given by the relation

$\mathrm{DoP}=\frac{\left[\left(\kappa^{2} \varepsilon_{D}^{4} \varepsilon_{M}^{4}-I_{R}^{4} I_{M}^{4} \Delta^{2}+\kappa^{2} I_{t}^{4}-\varepsilon_{C}^{4} \Delta^{2}\right)^{2}+4\left(I_{R}^{2} I_{M}^{2} \kappa \varepsilon_{D}^{2} \varepsilon_{M}^{2} \Delta+I_{t}^{2} \kappa \varepsilon_{C}^{2} \Delta\right)^{2}\right]^{1 / 2}}{\kappa^{2} \varepsilon_{D}^{4} \varepsilon_{M}^{4}+I_{R}^{4} I_{M}^{4} \Delta^{2}+\kappa^{2} I_{t}^{4}+\varepsilon_{C}^{4} \Delta^{2}}$.

Ideally, the PBS would block the entire TM component for transmission and it would not reflect any part of the TE component, which means $\varepsilon_{D}$ and $\varepsilon_{C}$ would be equal to zero. In this case, the second term would go to zero, while the first term's magnitude would depend on the balance between the two polarization components exiting the polarization transformer. However, the small TM transmitted component and TE reflected component in the PBSs cause the second term to be nonzero. Careful inspection of this term shows that there are no subtracted quantities. Therefore, the only way to compensate for its adverse effect is to find a way to leak these components out of the system before the beams recombine.

\section{Results and Discussion}

\subsection{Experiment Demonstration}

To test the performance of the depolarizer concept, a benchtop experiment was implemented with bulk optical elements. A schematic diagram of the setup is shown in Fig. 8. The PBS specifications for TE transmission $T_{p}$ and TM reflection $R_{s}$ are $T_{p}>96 \%$ and $R_{s}>98 \%$. The mirror reflectance is $R>99.8 \%$.

The optical source is a superluminescent diode (SLD) with an output power of $0.3 \mathrm{~mW}(-5.23 \mathrm{dBm})$. Figure 9(a) shows the SLD power spectrum measured with an optical spectrum analyzer. The center wavelength is approximately $1330 \mathrm{~nm}$ and the full width half maximum (FWHM) spectral width is $53.3 \mathrm{~nm}$. Thus, its coherence length is

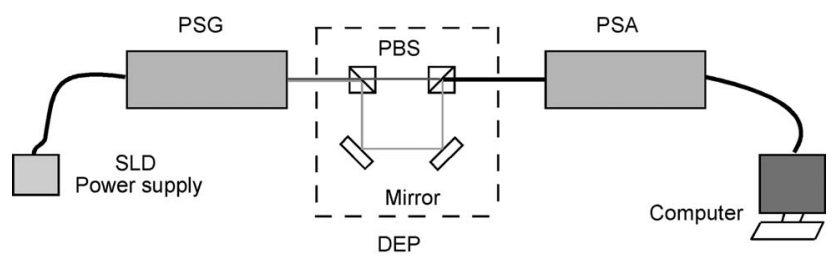

SLD---SuperLuminescent Diode,

PSG---Polarization State Generator, PSA---Polarization State Analyzer PBS---Polarization BeamSplitter, DEP---Depolarizer

Fig. 8 Schematic diagram of benchtop arrangement for the depolarizer. 


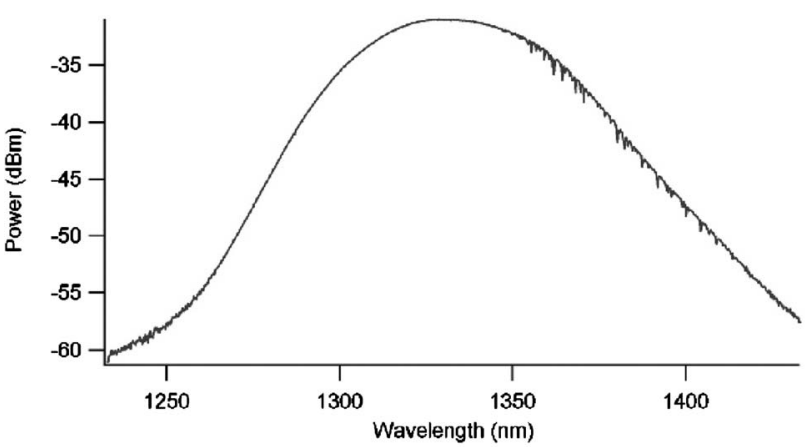

(a)

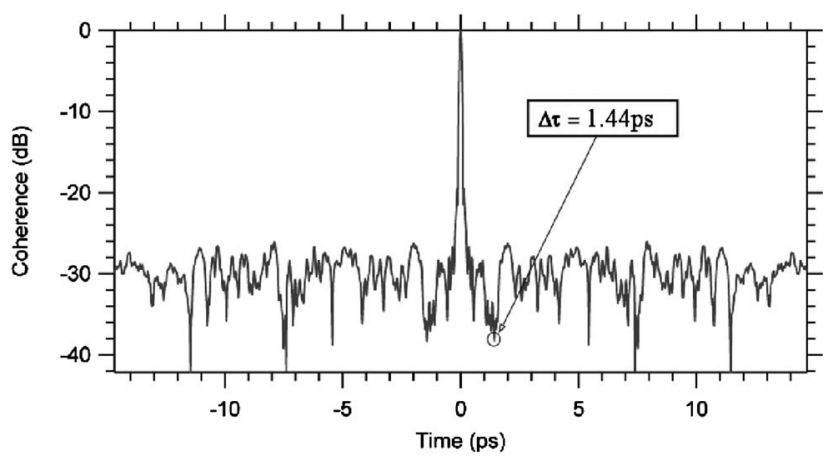

(b)

Fig. 9 (a) SLD's optical spectrum as measured by an ANDO 6315E spectrum analyzer and (b) coherence function calculated from the optical spectrum data. Note that the first minimum occurs at a time of $1.44 \mathrm{ps}$. This time delay corresponds to a path length difference of $265 \mu \mathrm{m}$ for the waveguide system discussed in Secs. 2.1 and 2.2.

$\sim 33 \mu \mathrm{m}$. The path difference between the reflection $(R)$ and transmission $(T)$ paths in the depolarizer is about $10 \mathrm{~cm}$, which is far greater than the coherence length of the source.

The depolarizer is mounted between the polarization state generator (PSG) and polarization state analyzer (PSA) of a Mueller matrix polarimeter to measure the polarization characteristics of the depolarizer. Accurate measurements depend upon careful alignment of the $R$ and $T$ beam paths to ensure colinearity of the output beams from each path.

To obtain equal intensity TE and TM components at the output of the depolarizer, the input polarization state to the depolarizer from the source is set to $48 \mathrm{deg}$ linear polarization to compensate for the small difference between $T_{p}$ and $R_{s}\left(T_{p}<R_{s}\right)$. The experiments were arranged to test three configurations: (1) $T$ path only, (2) $R$ path only, and (3) combined $T$ and $R$ paths. For the first two cases, we expect the DoP to be close to 1 , while for the third we expect the DoP to be very close to zero.

The experimental results are listed in Table 1. For the first two cases, the output DoP is very close to one, indicating that the light is highly polarized as expected. For the third case, the DoP is $0.002(-27.0 \mathrm{~dB})$, which means that the light has been well depolarized. Note that the insertion loss for the depolarizer is quite small at $0.079 \mathrm{~dB}$ and that the insertion loss for the $T$ only and $R$ only paths is $3 \mathrm{~dB}$, as one would expect.
Table 1 Measurement results of the depolarizer in bulk version.

\begin{tabular}{lcccc}
\hline \hline Configurations & $\begin{array}{c}\text { Output } \\
\text { DoP }\end{array}$ & $\begin{array}{c}\text { Out DoP } \\
(\mathrm{dB})\end{array}$ & Out $T$ & $\begin{array}{c}\text { Insertion } \\
\text { Loss }(\mathrm{dB})\end{array}$ \\
\hline$T$ only & 0.980 & -0.088 & 0.497 & 3.054 \\
$R$ only & 0.993 & -0.03 & 0.491 & 3.076 \\
$R$ and T & 0.002 & -27.0 & 0.982 & 0.079 \\
\hline \hline
\end{tabular}

Based on the experimental measurements, the Mueller matrix for this benchtop depolarizer ( $R$ and $T$ configuration) is

$\mathbf{M}_{R T}=\left[\begin{array}{cccc}0.982 & 0 & 0 & 0 \\ -0.002 & 0.957 & 0.104 & -0.006 \\ 0 & 0.0114 & 0.012 & 0 \\ 0.004 & 0 & 0 & 0\end{array}\right]$.

This is close to the ideal Mueller matrix for depolarizing a beam with equal TE and TM polarization components, which is given by

$\mathbf{M}_{R T}^{\text {ideal }}=\left[\begin{array}{llll}1 & 0 & 0 & 0 \\ 0 & 1 & 0 & 0 \\ 0 & 0 & 0 & 0 \\ 0 & 0 & 0 & 0\end{array}\right]$.

The upper left-hand element of the measured matrix $\mathbf{M}_{R T}$ is the total transmission for the depolarizer ( $T$ and $R$ configuration). The four numbers in the central $2 \times 2$ matrix,

$\left[\begin{array}{cc}0.957 & 0.104 \\ 0.0114 & 0.012\end{array}\right]$,

of $\mathbf{M}_{R T}$ represent a $3-\mathrm{deg}$ diattenuation for the $T$ only path. The other nonzero matrix elements are all very small and represent slight nonidealities of the depolarizer together with measurement noise. This experiment shows that a DoP as low as $0.002(-27 \mathrm{~dB})$ can be achieved with component properties, as already explained.

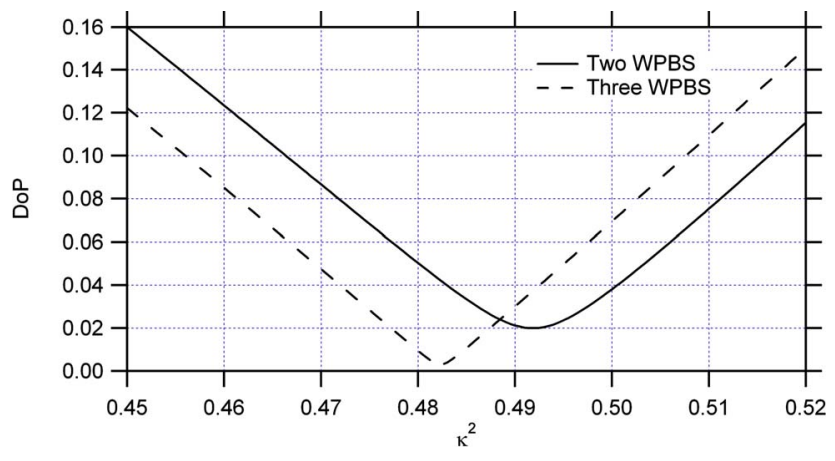

Fig. 10 DoP at the integrated depolarizer output as a function of the fraction of incident power with a TE polarization state on the depolarizer ring. 


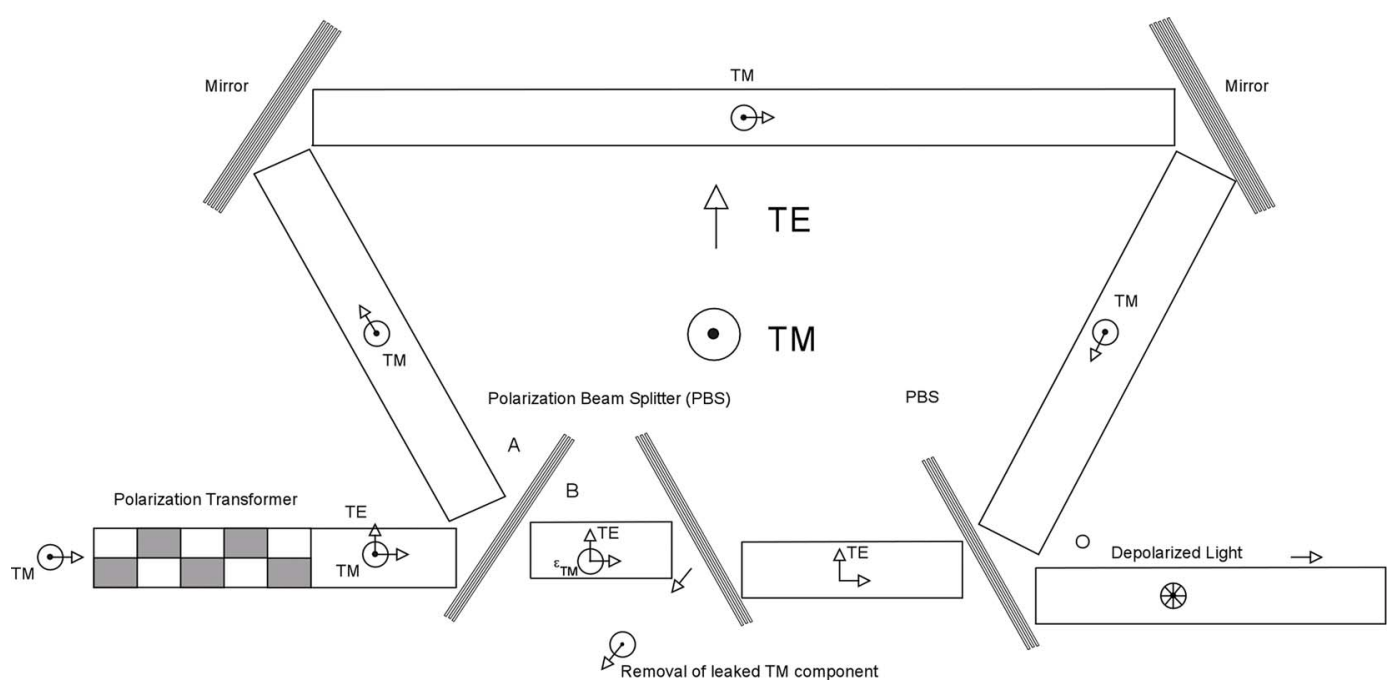

Fig. 11 Three-PBS depolarizing ring configuration. The middle PBS allows TE polarized light to be transmitted and significantly attenuates the unwanted TM polarization component that leaked through the first PBS by reflecting it away from the depolarizing ring.

\subsection{DoP Analysis of Integrated Optic Depolarizer}

The components for the integrated optic depolarizer (IOD) do not behave like ideal components. The mirrors have losses and the PBSs have a small amount of crosstalk. We can analyze how nonideal components will affect the DoP of light exiting the depolarizer by using the expression derived in Sec. 2.3.

Let's consider that the WPBS designed for the integrated depolarizer has $I_{R}^{2}=0.97, \varepsilon_{D}^{2}=0.03, I_{t}^{2}=0.96$, and $\varepsilon_{C}^{2}=0.04$. The mirrors have $I_{M}^{2}=0.99$ and $\varepsilon_{M}^{2}=0.09$. For these component parameters, the dependence of the DoP on the fraction of TE power coming out of the polarization rotator (see Fig. 4) is depicted in Fig. 10. Note that the range over which the DoP is less than 5\% is 47.9 to $50.5 \%$. This narrow range places a tight tolerance on the polarization transformer fabrication.

This design can be improved to achieve a lower DoP by adding a third WPBS, as shown in Fig. 11, which will leak much of the unwanted TM component out of the transmission path. The effect of the three-WPBS configuration is shown in the dashed curve of Fig. 10. By leaking out most of the unwanted TM component in the transmission path, the minimum DoP is reduced to less than $1 \%$.

This analysis shows the degradation of the DoP due to imperfections in the depolarizer's components. To achieve low DoPs, the intensities of both paths must be closely matched. While nonideal components will reduce the optical efficiency of the IOD, a low DoP is still achievable as long as the intensity in both paths is balanced. We showed that by improving this balance with three WPBSs, lower DoPs can be obtained.

\section{Conclusion}

We have presented an approach to create an integrated depolarizer for IFOG applications. The design is based on separating the TE and TM components of the incident waveguide mode and delaying the TM component with respect to the TE component past the coherence length, and then adding them to obtain depolarized light. We have shown how air trenches can be used to realize a WPBS and mirror. Finally, we analyzed the degradation of the depolarizer's performance due to nonideal components.

\section{Acknowledgement}

This work was supported in part by DARPA Grant N6600104-1-8933 and National Science Foundation Grant EPS0091853.

\section{References}

1. H. C. Lefevre, The Fiber-Optic Gyroscope, pp. 51-55, Artech House, Boston (1993).

2. K. Bohm, P. Marten, K. Petermann, E. Weidel, and R. Ulrich, "Low drift fibre gyro using a superluminescent diode," Electron. Lett. 17, $352-353$ (1983).

3. K. Bohm, K. Petermann, and E. Weidel, "Performance of Lyot depolarizers with birefringent single-mode fibers," J. Lightwave Technol. 1(1), 71-74 (1983).

4. D. Lutz, "A passive fiber-optic depolarizer," IEEE Photonics Technol. Lett. 4(4), 463-465 (1993).

5. M. Martinelli and J. C. Palais, "Dual fiber-ring depolarizer," J. Lightwave Technol. 19(6), 899-905 (2001)

6. K. Takada, K. Okamato, and J. Noda, "New fiber-optic depolarizer," J. Lightwave Technol. 4(2), 213-219 (1986).

7. S. Yamashita and K. Hotate, "Polarization-independent depolarizers for highly coherent light using Faraday rotator mirrors," J. Lightwave Technol. 15(5), 900-905 (1997).

8. E. Collett, Polarized Light: Fundamentals and Applications, Marcel Decker, New York (1993).

9. Y. Shani, R. Alferness, T. Koch, U. Koren, M. Oron, B. Miller, and M. Young, "Polarization rotation in asymmetric periodic loaded rib waveguides," Appl. Phys. Lett. 59, 1278-1280 (1991).

10. S. Obayya, B. Rahman, and H. El-Mikati, "Vector beam propagation analysis of polarization conversion in periodically loaded waveguides," IEEE Photonics Technol. Lett. 12(10), 1346-1348 (2000).

11. W. Huang and Z. Mao, "Polarization rotation in periodic loaded rib waveguides," J. Lightwave Technol. 10(12), 1825-1831 (1992).

12. J. Cai, J. Jiang, and G. P. Nordin, "Ultrashort waveguide polarization converter using a subwavelength grating," presented at Integrated Photonics Research, San Francisco (2004).

13. Photon Design, www.photond.com.

14. Max Born and Emil Wolf, Principles of Optics, 7th ed., Chap. 1, Cambridge University Press, (1999).

15. A. Taflove, Computational Electrodynamics: The Finite-Difference Time-Domain Method, Artech House, Boston (1995). 
Jianhua Jiang is a research scientist for Nano and Micro Devices Lab (NMDL) at the University of Alabama in Huntsville (UAH). He received his undergraduate degree in physics and his MS degree in optics from Tsinghua University, Beijing, China, in 1993 and 1996, respectively, and his $\mathrm{PhD}$ degree in optical science and engineering from the University of Alabama in Huntsville in 2000. His research interests are rigorous grating theory and numerical modeling methods for photonic devices.
Biographies and photographs of other authors not available. 